\title{
Fruit Detection using Improved Multiple Features based Algorithm
}

\author{
Hetal N. Patel \\ Assistant Professor \\ Electronics and Communication \\ Engg. Department. \\ A.D.Patel Insitute of \\ Technology, \\ New V.V.Nagar, Gujarat, India
}

\author{
Dr. R.K.Jain \\ Principal, \\ A.D.Patel Insitute of \\ Technology, \\ New V.V.Nagar, Gujarat, India
}

\author{
Dr. M.V.Joshi \\ Professor, \\ Electronics Engg. Department, \\ Dhirubhai Ambani Institute of \\ Information and \\ Communication Technology, \\ Gandhinagar, Gujarat, India
}

\begin{abstract}
Efficient locating the fruit on the tree is one of the major requirements for the fruit harvesting system. This paper presents the fruit detection using improved multiple features based algorithm. To detect the fruit, an image processing algorithm is trained for efficient feature extraction. The algorithm is designed with the aim of calculating different weights for features like intensity, color, orientation and edge of the input test image. The weights of different features represent the approximate locations of the fruit within an image. The Detection Efficiency is achieved up to $90 \%$ for different fruit image on tree, captured at different positions. The input images are the section of tree image. The proposed approach can be applied for targeting fruits for robotic fruit harvesting.
\end{abstract}

Keywords: Fruit harvesting system, Multiple features, Weight of features.

\section{INTRODUCTION}

Fruit detection system is primarily developed for robotic fruit harvesting. However this technology can easily be tailored for other applications such as on tree yield monitoring, crop health status monitoring, disease detection, maturity detection and other operations which require vision as a sensor. For fruit harvesting system, it is very necessary to detect the fruit on the tree more efficiently. The vision based fruit harvesting system for the fruit detection basically depend on the contribution of different features in the image. The four basic features which characterize the fruit are: intensity, color, edge and orientation. This paper proposes an efficient multiple features based algorithm for the fruit detection on tree. Color features in image could be successfully used to segment defects on 'Jonagold' apples are demonstrated in [8]. Texture features are found to contain useful information for quality evaluation of fruit and vegetables, e.g., classification of grade of apples after dehydration with the accuracy of 95\% [9]. Color and texture features are used to locate green and red apples [10]. Combining many features and classifiers, where all features are concatenated and fed independently to each classification algorithm. The fusion approach is validated using the multi-class fruit-vegetable categorization task in a semi-controlled environment, such as a distribution center or the supermarket cashier. The results show that the solution is able to reduce the classification error up to $15 \%$ point with respect to the baseline [1]. The stereoscopic vision system of tomato-harvesting robot with a detection accuracy of approximately $85 \%$ is achieved in [6]. The author in [11] use the local or shape based analysis to detect the fruit that allows for rapid detection and were able to detect the fruit at specific maturity stages i.e., fruit with a color different from the background. The models for illumination and surface reflectance for use in outdoor color vision, and in particular for predicting the color of surfaces under outdoor conditions is discussed in [12]. The authors in [13] have developed the fruit detection algorithm with the combined multiple viewing techniques for a tree canopy. The color and texture features are used for the color recognition. An efficient fusion of color and texture is used for fruit recognition. The recognition is done by minimum distance classifier based upon the statistical and co-occurrence features derived from the wavelet transformed sub-bands [4].The spherical fruit recognition system developed with a laser range-finder model and a dual color/shape analysis algorithm in [17]. For the analysis of the image of oil palm fresh fruit bunches (FFB), the development of outdoor image analysis of oil palm fruit fresh bunches (FFB) are required. The software analysis generates the mathematical model and correlation factor between the light intensity in relation to value of FFB from Red, Green and Blue component of image taken [3]. In citrus manufacturing industries, caliper and color are successfully used for the automatic classification of fruits using vision systems. A computer vision system capable of detecting defects in the citrus peel and also classifying the type of flaw is presented in [5]. The on-line estimation of the quality of oranges, peaches and apples the evaluation of the efficiency of these techniques regarding the following quality attributes hereby size, color, stem location and detection of external blemishes is presented in [7]. The segmentation procedure is used based on a Bayesian discriminate analysis, allowed fruits to be precisely distinguished from the background. Fruit recognition algorithm consists of segmentation, region labeling, filtering, perimeter extraction and perimeter-based detection. In [15] for the segmentation of the orange fruit, the enhancement was done using the red chromaticity coefficient which enabled adaptive segmentation under variable outdoor illumination. Two image fusion approaches: Laplacian pyramid transform (LPT) and fuzzy logic are applied for improving the fruit detection efficiency as compared to the fruit detection using only thermal image in [16]. A machine vision algorithm consists of segmentation, region labeling, size filtering, perimeter extraction and perimeter-based detection, for the recognition of orange fruit is presented in [21]. Our work in this paper presents a fruit detection using multiple features based algorithm. A simple feature can not entirely represent the character of the fruit region. Therefore, multiple features analysis is used in the proposed method. For efficient detection of fruit on tree, various types of features like color, intensity, edge and orientation are used. The computed features are then integrated according to their weights. After integrating the feature map, then we obtain the final image map. According to the feature map, the fruit is accurately 
located within an image. Because of the use of multiple features and efficient feature integration, the proposed method gives good result of detection in clustered region of the test image. The paper is structured as follows: the next section discuss the Proposed Method. Section 3 gives the detection Results and discussion. Finally, in Section 4 conclusion of the proposed approach are presented.

\section{PROPOSED METHOD FOR FRUIT DETECTION}

First stage of fruit detection is to extract the features like intensity, color, edge and orientation. These extracted features are integrated using weights according to their different effects on the image region [18]. The integrated map is segmented using global thresholding for generating the binary map. Finally according to the binary image, the fruit regions are extracted.

\subsection{Extraction of Intensity and Color Features}

There are many color models based on human color perception. The on tree fruit image is converted into HSV color model space. Such models want to divide color into a set of coordinates de-correlating human impressions such as hue, saturation, and intensity. The generated $\mathrm{H}$ and $\mathrm{S}$ channel data is used to represent the color features of the input image and I channel data is used to represent the intensity features of the input image.

\subsection{Extraction of Orientation Features}

The orientation feature map are obtained by filtering the intensity feature map using four Gabor filters with the orientation $0^{\circ}, 45^{\circ}, 90^{\circ}, 135^{\circ}$ respectively. The elliptical Gabor filter is defined using equation (1)

$G(x, y)=g\left(x^{\prime}, y^{\prime}\right) \exp \left(2 \pi j F \sqrt{\left.x^{2}+y^{2}\right)}\right.$

where, $\left(x^{\prime}, y^{\prime}\right)=(x \cos \theta+y \sin \theta,-x \sin \theta+y \cos \theta)$

where, $F$ is the spatial central frequency of the filter in the frequency domain, $g(x, y)$ is 2-D Gaussian envelope calculated using equation (2).

$g(x, y)=\left(\frac{1}{2 \pi \sigma_{x} \sigma_{y}}\right) \exp \left[-\frac{1}{2}\left(\frac{x^{2}}{\sigma_{x}^{2}}+\frac{y^{2}}{\sigma_{y}^{2}}\right)\right]$

Here $\sigma_{x}$ and $\sigma_{y}$ are variances (scale factors) along $\mathrm{x}$ and $\mathrm{y}$ axes respectively. $(x, y)$ is the center of the receptive field in the spatial domain, $\theta$ is the rotation angle of the filter.

\subsection{Extraction of Edge Features}

The edge features refers to the process of identifying and locating sharp discontinuities in the image. The discontinuities are abrupt changes in pixel intensity which characterize boundaries of the fruits in a scene. The gradient points in the direction of most rapid increase in intensity. The gradient magnitude is used as a measure of edge features.

\subsection{Extraction of Fruit Region from Feature Maps}

After extracting the low-level visual features, in the form of intensity, color, edge and orientation features. The integrated feature maps are computed. The following steps are to be performed for extracting the fruit region from different features maps:
Step 1: Reconstruct the image with phase values of frequency spectrum, to eliminate the influence of magnitude spectrum and get the region of interest. The local points of the fruit region of different feature maps like intensity, color, edge and orientation, can be computed using equation (3).

$F_{\text {local }}(x, y)=\frac{1}{M * N} \sum_{u=1}^{M} \sum_{v-1}^{N} P(u, v) e^{\frac{-j 2 \pi u x}{M}} e^{\frac{-j 2 \pi v y}{N}}$

Where, $\mathrm{P}(\mathrm{u}, \mathrm{v})$ is the phase spectrum of the feature map.

Step 2: Compute the global points of the fruit region for different feature maps using equation (4). The result is the points where the fruit region is most probably located.

$F_{\text {global }}(x, y)=e^{\frac{a b s\left(f(x, y)-f_{\text {avg }}(x, y)\right.}{f_{\text {avg }}(x, y)}}$

where, $f_{\operatorname{avg}(x, y)}$ is the average value of the feature map.

Step 3: The area have novel and rare feature values, the novel feature values were computed using above two steps, the rare feature values are computed using the histogram of the feature map [18]. The rare points of the fruit region for different feature maps are computed using equation (5).

$F_{\text {rare }}(x, y)=\frac{1}{\operatorname{hist}(f(x, y))}$

where, $f(x, y)$ is the feature value at $(x, y)$ and $h(*)$ is the histogram of the feature map.

Step 4: Finally, local, global and rare feature points need to be combined into a feature conspicuity map. The weights of each feature points are calculated using the variance of each result. The higher the variance is the more important the feature points are. According to the weights the feature conspicuity map can be generated using equation (6).

$\left\{\begin{aligned} v & =\operatorname{variance}(f(x, y)) \\ w_{i} & =\frac{v_{i}}{\sum_{i=1}^{3} v_{i}} \\ F_{c} & =w_{1} * F_{\text {local }}+w_{2} * F_{\text {global }}+w_{3} * F_{\text {rare }}\end{aligned}\right.$

Step 5: The feature conspicuity maps are extracted using step 1 to step 4 for intensity, color, edge and orientation features of input image separately. These regions are now required to integrate accurately. We have used a dynamic feature integration strategy. The feature points of intensity, color, edge and orientation features are now used for calculating their weights, for which a global thresholding is used. The threshold $T$ can be computed using equation (7). $T=\frac{1}{M * N} \sum_{i=1}^{M} \sum_{j=1}^{N} f(i, j) \quad \ldots \quad \ldots$

Where, $\mathrm{T}$ is the Threshold, $f(i, j)$ is the feature conspicuity map of the size $M * N$.

$>$ In the feature conspicuity map, the points, whose values are higher than the Threshold, are considered as useful points $\left(\mathrm{W}_{\mathrm{A}}\right)$.

$>$ Compute the average distance between the useful points computed above and the image center as the location criterion for all point $\left(\mathrm{W}_{\mathrm{L}}\right)$.

$>$ If the useful points are not cluster together but distribute separately in the feature map, the distribution of useful points are needed to calculate. The distribution of useful points within an image is calculated by computing the distance function 
between the useful points and the centroid of the cluster $\left(\mathrm{W}_{\mathrm{D}}\right)$ [18].

$>$ Compute the integrated feature map using equation (8).

$$
\left\{\begin{array}{l}
W_{i}=\frac{\frac{1}{W_{\text {final }}^{i}}}{\sum_{i=1}^{m} W_{\text {final }}} \\
W_{\text {final }}^{i}=W_{A}^{i}+W_{L}^{i}+W_{D}^{i}
\end{array}\right.
$$

Where, Wi means the weight of feature conspicuity map and $m$ is the number of feature conspicuity maps (here $m=4$ ).

Step 6: According to the weights calculated in step 5, the final fruit region is calculated using equation (5).

$F R_{\text {map }}=\sum_{i=1}^{4} W i * F_{C i} \quad \ldots \quad \ldots$

Where, $F R_{\text {map }}$ is the final fruit region and $F c i$ means feature conspicuity map of intensity, color, edge and orientation features respectively.

\subsection{Fruit Detection}

The extracted Fruit Region is now used for generating the binary mask by comparing each pixel values with the global Threshold of Fruit Region. The Binary Mask is calculated using equation (6).

$$
\text { Bmask }=\left\{\begin{array}{l}
1, F R_{\text {map }} \geq T \\
0, F R_{\text {map }}<T
\end{array}\right.
$$

\section{DETECTION RESULTS}

The proposed fruit detection algorithm was extensively tested with many tree images to ensure proper detection of fruits from an image. We have collected many images from image search engines and selected more than 100 images in which each one contains one or more fruit regions. This section presents detection results and analysis of the proposed fruit region detection approach. The proposed fruit region detection method has been tested on a computer with Intel Atom $1.66 \mathrm{GHz}$ and $1 \mathrm{~GB}$ RAM. Figure 1 shows the simulation results of the fruit detection algorithm.

\subsection{Discussion}

If the input tree image contains one or more fruit regions, the proposed method efficiently detect the fruits. As the color features of the fruit region in the tree image are different than the background, the proposed method gives good result of detection. If the input image is very complex and do not contain a different color fruits, the proposed method fails to extract the fruit regions which is shown in last row of fig 2 . Fig 2 shows the simulation results of the proposed method. The fig 2(a) shows the input image. In the second column i.e. fig 2(b), we show the integrated feature map and the third column, fig2(c) shows the Binary Map. The detected fruit region is shown in fig $2(\mathrm{~d})$.

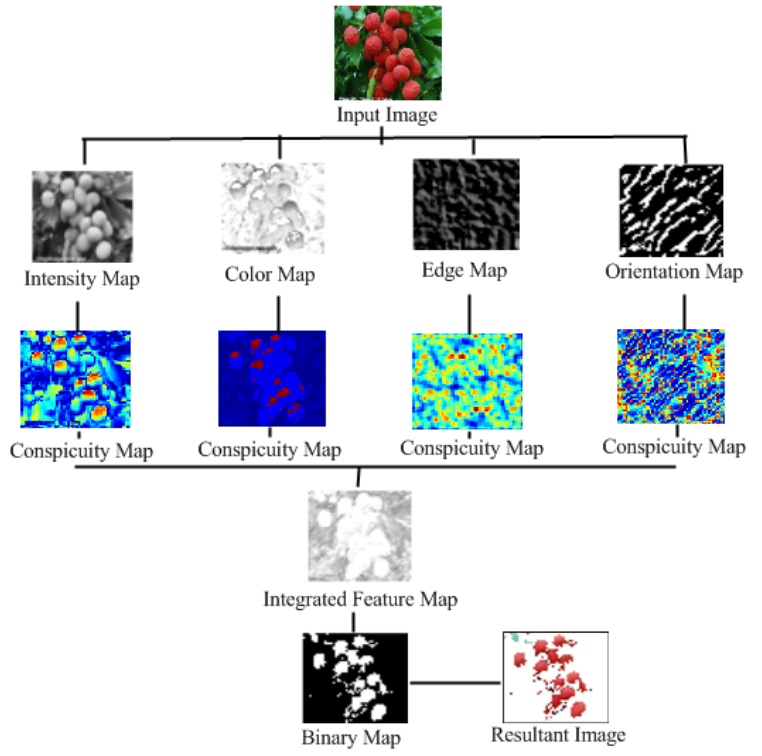

Figure 1: Simulation Results of Fruit Detection Algorithm

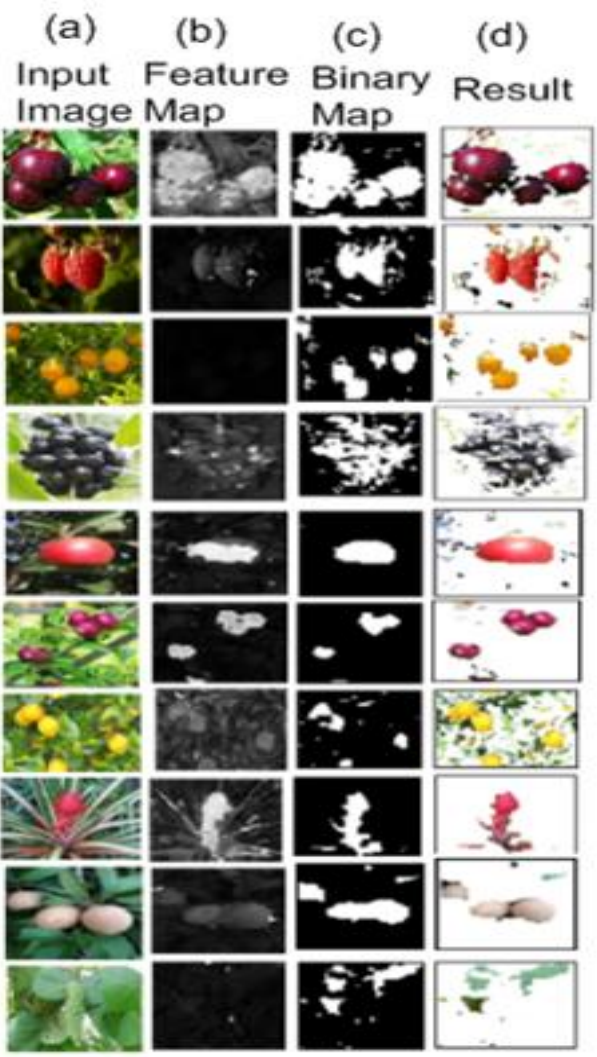

Figure 2: Simulation Results

Our results indicate more than $95 \%$ of successful extraction of fruit region from the background. The proper selection of threshold plays a major role in successful extraction of fruit region. If the Threshold is too high not all the pixels of the fruit region will be extracted. A low threshold leads to too many pixels be extracted. . A global threshold is selected for making balancing between the true positives and low false negatives. Few more simulation results are shown in fig 3 . The input image is shown in fig 3(a). The integrated feature 
map is shown in fig 3(b) and the final detected fruit region is shown in fig 3(c).

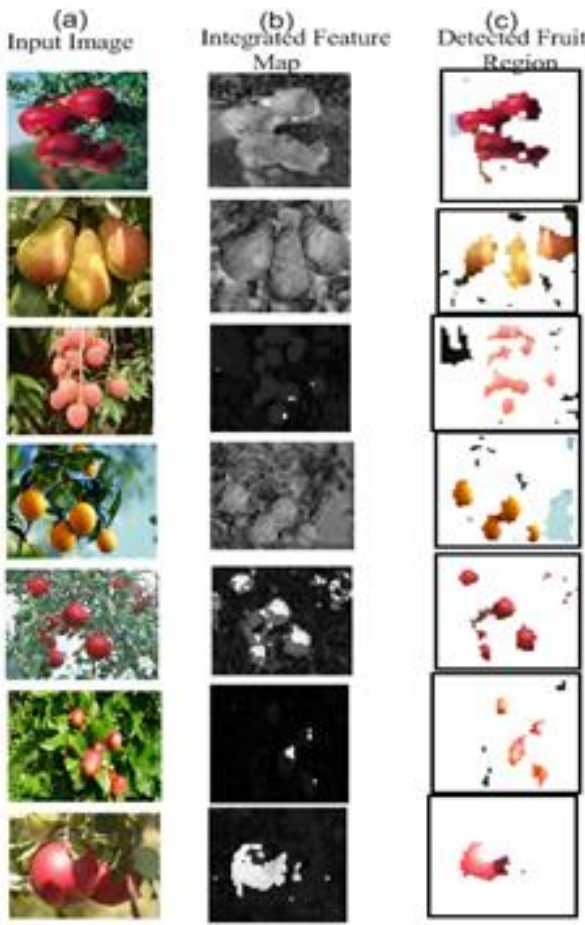

Figure 3: Fruit Detection Results

We have developed image processing technique using multiple features for fruit detection. The proposed technique is robust under complex and clustered background. The Fig 4 shows the comparison of different binary map using individual intensity, color, edge and orientation features versus integrated feature map. The binary map generated using only intensity detects the half region of fruit while detecting some background also. Similarly if we consider only orientation feature than some part of the object is not detected in the final image. So, the feature points are integrated according to their weights for generating more efficient binary map for fruit detection. The weights of each feature maps are also shown in the fig 4 .

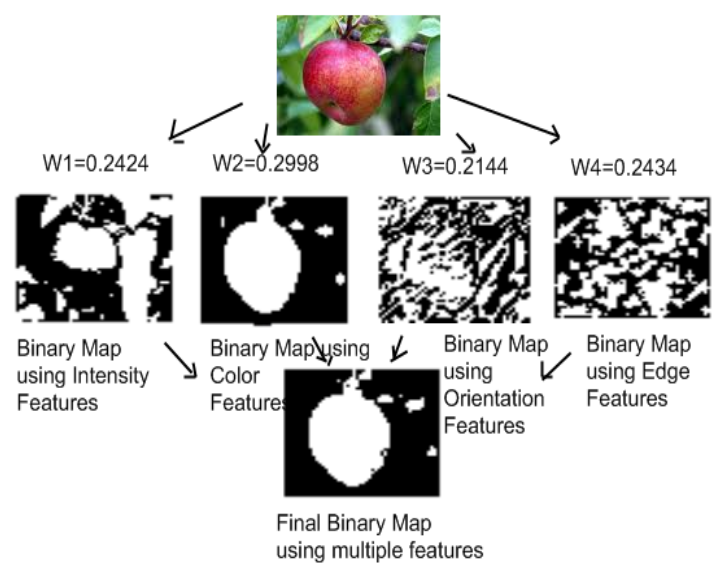

Figure 4: Comparision between multiple feature map and single feature map.

The efficiency of the fruit detection algorithm depends on the features and their weights calculated for an input image.

\subsection{Comparison}

Orange fruit detection by Hannan [21] is based on the chromaticity value of the fruit image. The detection of fruit is depending on the threshold of r-histogram. The threshold of $r$ histogram is defined manually for the orange fruit. While in our proposed method; there is no need of any manual change. We have developed the fruit detection algorithm for different type of fruits. The proposed method is not domain-specific and does not impose limits on the variety of sectional fruit images.

\section{CONCLUSION}

Efficient fruit detection using multiple feature based algorithm is developed and proposed in this paper. Multiple features like intensity, color, edge and orientation are analyzed. It computes the feature map for different type of feature points and according to the feature map the fruit regions are extracted. The process is entirely automatic and does not need user intervention. The proposed method is not domain-specific and does not impose limits on the variety of clustered sectional tree image. It can be used for all kind of images provided that there are at least one or more meaningful fruit regions. A simple feature can not entirely represent the character of the fruit region. Therefore, multiple features analysis is used in the proposed method. In this paper, authors consider colors, intensity, edge and orientations as the features of the image. However, it is very likely that there are some other features such as symmetry features also should be considered. What features and how many features should be extracted according to the target will also be included in the future work. Fruit detection using other imaging devices other than the usual RGB camera will also be investigated in the future work.

\section{REFERENCES}

[1] Anderson Rocha, Daniel C. Hauagge, Jacques Wainer, Siome Goldenstein, "Automatic fruit and vegetable classification from images", Computers and Electronics in Agriculture, Vol. 70, pp. 96-104, 2010.

[2] Arivazhagan S., Newlin Shebiah R., Nidhyanandhan Selva S., Ganesan L., " Fruit Recognition using Color and Texture Features", Journal of Emerging Trends in Computing and Information Sciences, vol. 1, no. 2, Pages: 90-94, Oct 2010.

[3] Ismail Wan Ishak Wan, Razali Mohd. Hudzari, "Outdoor colour recognition system for oil palm fresh fruit bunches (FFB)", International Journal of Machine Intelligence, Vol-2, Issue 1, Pages:01-10, 2010.

[4] Woo Chaw Seng and Seyed Hadi Mirisaee, "A New Method for Fruits Recognition System", MNCC Transactions on ICT, Vol. 1, No. 1, June 2009.

[5] Lopez Jose J., Cobos Maximo and Aguilera Emanuel, "Computer-based detection and classification of flaws in citrus fruits", Internation conference on natural image processing (ICONIP-2009).

[6] Fernández, L., Castillero, C. and Aguilera, J. M., "An application of image analysis to dehydration of apple discs" Journal of Food Engineering, vol.67, pp.185-193, 2005.

[7] Zhao, J.T., J. Katupitiya, J., "On-tree fruit recognition using texture properties and color data", International conference on Intelligent Robots and Systems, pp. 263268, 2005. 
[8] Leemans, V. and Destain, M.-F, "A real-time grading method of apple based on features extracted from defects" Journal of Food Engineering, vol.61, pp.83-89, 2004.

[9] Hayashi Shigehiko, Ota Tomohiko, Kubota Kotaro, Ganno Katsunobu and Kondo Naoshi, "Robotic Harvesting Technology for Fruit Vegetables in Protected Horticultural Production", Information and Technology for Sustainable Fruit and Vegetable Production FRUTIC 05, France.

[10] Blasco J., Aleixos N., Molto E., " Machine Vision System for Automatic Quality Grading of Fruit", Biosystems Engineering, Vol-85, Issue 4, Pages-415423, August 2003.

[11] Jimenez A.R., Ceres R., Pons J.L., "A Survey of Computer Vision Methods for Locating Fruit on Trees", Transaction of the ASAE, Vol. 43(6), pages: 1911-1920, 2000.

[12] Shasi Buluswar (2002), "Models for Outdoor Color Vision", Doctoral dissertation, University of Massachusetts, Amherst.

[13] Bulanon D.M., Burks T.F., Alcahnatis V., "Improving Fruit detection for robotic fruit harvesting", ISHS Acta Horticulturae 824: Internation Symosium on Application of Precision Agriculture for Fruits and Vegetables.

[14] Jimenez R., Jain A.K., Ceres R., Pons J.L., "Automatic fruit recognition: A survey and new results using Range/Attenuation images", Pattern Recognition, 32 (10), pp. 1719-1736, 1999.
[15] Hannan M.W., Burks T.F., Bulanon D.M., "A Machine Vision Algorithm for Orange Fruit Detection", Agricultural Engineering International: the CIGR E journal, vol-XI,Pages:1-7, December-2009.

[16] Bulanon D.M., Burks T.F., Alchanatis V., " Image Fusion of visible and thermal images for fruit detection", Biosystems Engineering, Vol-103, Issue-1, May 2009, pages:12-22.

[17] Bulanon D.M., Burks T.F. and Alchanatis V., " Study of temporal variation in citrus canopy using thermal imaging for citrus fruit detection", Biosystems Engineering, Vol-101, Issue 2, Pages 161-171, October 2008.

[18] Qiaorong Zhang, Haibo Liu, Jing Shen, Guochang Gu, Huimin Xiao, "An Improved Computational Approach for Salient Region Detection.” ,Journal of Computers, vol.5,no-7, pp.1011-1018, July 2010.

[19] Itti L, Kouch C, "Feature combination strategies for saliency-based visual attention systems," Journal of Electronic Imaging, vol. 10, pp.161-169, January 2003.

[20] Zheshen Wang, Baoxin Li, "A Two-Stage Approach to Saliency Detection in Images," IEEE ICASSP 2008, April 2008, pp. 965-96.

[21] Hannan M.W., Burks T.F., Bulanon D.M., "A Machine Vision Algorithm for Orange Fruit Detection", Agricultural Engineering International: the CIGR Ejournal, Vol-XI, December 2009. 